\title{
A MICRO THIN LAYER CHROMATOGRAPHY TECHNIQUE FOR URINARY AND SERUM AMINO ACIDS USING FLUORESCAMINE AS A STAINING REAGENT
}

\author{
Tso-Ren WAng, ${ }^{*}$ Ming-liang LeE, ${ }^{* *}$ and David A. Toke \\ Division of Medical Genetics, Department of Pediatrics, University of \\ Medicine \& Dentistry of New Jersey, Rutgers Medical School. \\ New Brunswick, New Jersey 08903, USA
}

\begin{abstract}
Summary A rapid, sensitive, inexpensive micro technique for thin layer chromatography of amino acids from urine and blood is described. The method which requires only $0.1 \mu 1$ of sample, uses a fluorescent reagent, fluorescamine, as a staining agent, and commercially available cellulose plates, Polygram Cel 300, as a supporting matrix. The great majority of amino acids can be detected when concentration is greater than $0.25 \mathrm{~mm}$. The application of this technique toward the identification of various abnormal amino acid metabolisms is discussed.
\end{abstract}

A method of deproteinization of serum, modified from a previously published technique, is also reported.

\section{INTRODUCTION}

The outcome of many of the inborn errors of amino acid metabolism, if undetected and untreated, is frequently severe mental retardation. Although in the past decade many countries have adapted mandatory PKU screening, screening programs for abnormal metabolism of amino acids other than phenylalanine have thus far received limited acceptance, mainly due to unfavorable cost/benefit ratios.

Theoretically, it seems possible to develop a single yet general method for the detection of any abnormal amino acid metabolism, as they all share a common reactive amino group. The method, based on this principle utilizes paper chromatography technique with ninhydrin as a staining reagent and as such, has been recommended by many laboratories (Thomas and Howell, 1973; Kelly, 1977). The

Received May 1, 1983

Abbreviations: PKU, phenylketonuria; MSUD, maple syrup urine disease; NHT, neonatal hypertyrosinemia; TEA, triethylamine.

* Present address: Department of Pediatrics, College of Medicine, National Taiwan University, Taipei, Taiwan.

** To whom correspondence should be addressed. 
method, however is rather laborious and takes a considerable amount of time to perform, thus rendering it a less than ideal candidate for screening purposes.

Recently, a very sensitive fluorogenic reagent, fluorescamine, (4-phenylspiro(furan-2(3H), 1'-phthalan)-3,3'-dione) was introduced by Udenfriend and co-workers (Udenfriend et al., 1972; Felix and Jimenez, 1974; Stein et al., 1973) which has been extensively used in the detection of amino acids and peptides from various sources. The reagent reacts with primary amines very rapidly (alkaline $\mathrm{pH}$, at room temperature). It can detect levels of primary amines of as low as $1 \mathrm{nmol} / \mathrm{ml}$ concentration. Another advantage of this reagent is that while the reagent will produce a fluorescent compound when coupled with amino acid, the reagent itself or its degradation product does not fluoresce (Udenfriend et al., 1972). Furthermore, the presence of ammonia, which is a normal component of urine and serum, produces relatively little fluorescence (Lai, 1977). Thus, we took advantage of this fluorescent reagent and have developed a micro technique for thin layer chromatography and attempted to use this as a general reagent for detection of amino acids. This report describes our initial results and shows that at least for those more frequently seen amino acids, with proper choice of supporting matrix for chromatography, a very rapid, inexpensive method can be derived.

\section{MATERIALS}

Samples. Urine: Urine samples were obtained directly from patients known to have PKU (treated or untreated), cystinuria or homocystinuria. Additionally, "abnormal" urine samples were artificially made by the addition of an individual amino acid (but not its metabolites) to the urine representing a final concentration of approximately the midpoint of the range observed in the corresponding disease (Table 1) (Bremer et al., 1981).

Because only a minute amount of urine is needed, it is possible for a very small amount of urine to be collected. Thus, the amino acid concentration can be falsely increased due to decrease of urine volume by evaporation. Therefore, it is advisable to apply the sample to the plate as soon as possible, or caution be made to avoid urine concentration.

Blood: Serum with or without deproteinization can be used (see below).

Thin layer plate. Plastic-backed cellulose thin layer plate, Polygram Cel 300 (Machery-Magel \& Co., Germany, distributed by Brinkman Instruments, Inc., Westbury, NY, USA) was presoaked in the chromatographic solvent (see below) at room temperature for at least $30 \mathrm{~min}$ with occasional shaking before use. This procedure is essential in obtaining a clear plate with fine resolution. The plate was cut into pieces of width of $2.5 \mathrm{~cm}$ to $7.5 \mathrm{~cm}$ and length of $6 \mathrm{~cm}$ depending on the number of samples to be tested (a $7.5 \mathrm{~cm} \times 6.0 \mathrm{~cm}$ plate can accomodate 10 samples). It is advisable to scrape off cellulose 1-2 mm from each side border of plate, to avoid uneven solvent front. The used plates can be regenerated by soaking in the solvent 
Table 1. Urinary amino acid concentrations. 2

\begin{tabular}{|c|c|c|c|c|c|c|}
\hline \multirow[b]{2}{*}{ Disease } & \multirow[b]{2}{*}{ Amino acid } & \multicolumn{3}{|c|}{ Normal (mM) } & \multirow{2}{*}{$\begin{array}{l}\text { Range in } \\
\text { diseases }\end{array}$} & \multirow{2}{*}{$\begin{array}{l}\text { Conc. used } \\
\text { in this } \\
\text { study } \\
\text { (mM) }\end{array}$} \\
\hline & & Neonate & $\begin{array}{c}\text { Child } \\
\text { (3-12 yr) }\end{array}$ & Adult & & \\
\hline \multirow[t]{3}{*}{ MSUD } & Leu & $0-.028$ & $.028-.111$ & $.002-.068$ & $.890-3.73$ & 2.0 \\
\hline & Ile & $0-.042$ & $.032-.077$ & $.006-.055$ & $.420-1.34$ & 1.0 \\
\hline & Val & $0-.067$ & $.020-.068$ & $.014-.053$ & $.595-3.55$ & 2.0 \\
\hline PKU, (Type I) & Phe & $0-.223$ & $.032-.141$ & $.015-.081$ & up to 5.5 & 3.0 \\
\hline NTN & Tyr & $0-.295$ & $.053-.225$ & $.008-.207$ & up to 6.90 & 4.0 \\
\hline \multicolumn{7}{|l|}{ Hyperglycinemia } \\
\hline Ketotic & Gly & $.375-3.45$ & $.379-1.89$ & $.229-2.08$ & up to 8.89 & 5.0 \\
\hline Non-ketotic & Gly & $.375-3.45$ & $.379-1.89$ & $.229-2.08$ & up to 300 & 15.0 \\
\hline Homocystinuria & Homocystine & trace & trace & trace & $2.29-4.30$ & 3.0 \\
\hline Histidinemia & His & $.068-1.18$ & $.395-1.71$ & $.425-1.86$ & up to 4.58 & 3.0 \\
\hline \multirow[t]{3}{*}{ Cystinuria } & Cystine & $0-.118$ & $.028-.172$ & $.017-.083$ & $1.17-2.70$ & 1.5 \\
\hline & Arg & $0-.030$ & trace & $0-.051$ & $.272-5.45$ & 3.5 \\
\hline & Lys & $0-.737$ & $.085-.857$ & $.077-1.12$ & $3.11-10.9$ & 5.0 \\
\hline
\end{tabular}

a Data adapted from those of Bremer et al., 1981.

as described above and can be used for approximately 3-5 times (then the spots become diffused with additional streaking).

Reagents. UV spectrophotometric grade acetone (water content, $0.04 \%$ ) and cation exchange resin, Dowex 50W-X8, $\mathrm{H}^{+}, 20-50$ Mesh, were purchased from J.P. Baker Chemical Co., Phillipsburg, NJ, USA. Fluorescamine, from Hoffman LaRoche Co., Nutley, NJ, USA, was prepared in $25 \mathrm{mg}$ per $100 \mathrm{cc}$ of acetone and stored in a dark bottle. Triethylamine (TEA) was obtained from Pierce Chemical Co., Rockford, IIl., USA, and was prepared fresh weekly in a solution in acetone and stored in a dark bottle. Amino acids were purchased from Sigma Chemical Co., St. Louis, MO., USA. Microcaps were obtained from Drummond Scientific Co. (distributed through Ace Scientific Supply Co., Linden, NI, USA). Spectrophotometric grade butanol and acetic acid were obtained from Fisher Chemical Co., Springfield, NJ, USA. $\left[{ }^{14} \mathrm{C}\right]$ Amino acids were obtained from Research Products, International Corp., Mt. Prospect, Ill., USA.

Chromatography. No special chromatographic vessel is needed. An ordinary 100-200 cc beaker or jar, depending on the size of the plate, is sufficient for chromatography. The solvent was the standard butanol-acetic acid-water mixture (12 : $3: 5$, in volume). 


\section{METHODS}

All the procedures were performed at room temperature.

Deproteinization of serum. Proteins were removed by cation exchange resin as originally reported by Ambler (1972). The following modifications were made: to $0.5 \mathrm{ml}$ of serum, $100-150 \mathrm{mg}$ of Dowex in acidic form (directly from bottle) was added and mixed occasionally for $20 \mathrm{~min}$. The supernatant was discarded. Dowex beads were then washed with $2 \mathrm{ml}$ of water 3 times (each takes less than $1 \mathrm{~min}$ ). The $\mathrm{pH}$ of the last wash is about 6. Amino acids were then eluted with $100 \mu \mathrm{l}$ of $5 \mathrm{M} \mathrm{NH}_{4} \mathrm{OH}$ for $40 \mathrm{~min}$ with occasional shaking. The amount of serum can be scaled down 5 times with all the others proportionally reduced. In the experiments where recovery of amino acids is needed, known amount (cpm) of undiluted radioactive amino acid $\left(5-10 \mu 1,1-2 \times 10^{5} \mathrm{cpm}\right)$ was added to the serum before the experiment. At each time interval, an aliquot was removed for radioactivity determination.

Deproteinization of urine, if necessary (see below), can be achieved in a similar fashion.

Application of samples. Spot $0.1 \mu$ of urine or serum (deproteinized or not) through a $0.5 \mu \mathrm{l}$ microcap, on a cellulose plate, $0.5 \mathrm{~cm}$ apart, $0.5 \mathrm{~cm}$ from the bottom, allow the spots to air dry. As a reference spot for the chromatography, standard amino acid mixture $(0.1 \mu \mathrm{l}$ of $5 \mathrm{~mm}$ each of Leu, Phe, Met, Tyr, Pro, Gly and His) was spotted at the center of the line of origin. Simultaneous chromatography with known concentration of known amino acids (above standard mixture) is important not only for position reference but also for internal control of staining.

Chromatography. A chromatographic jar or simple laboratory beaker, containing enough fluid to cover the bottom of about $0.3 \mathrm{~cm}$ is covered with aluminum foil. No equilibrium of the system is necessary. The solvent was allowed to ascend for $5 \mathrm{~cm}$ (about 30-32 $\mathrm{min}$ ), and the plate was removed and dried with warm air.

Staining. The staining is based on the procedure described by Lai (1977) with minor modifications. After chromatography, the plate was dipped through or rinsed with acetone for a few seconds and dried by air. This procedure is repeated once. The plate was then dipped in a $1 \%$ TEA solution for 5 min and then allowed to air dry. Before the plate was completely dried, it was dipped in a fluorescamine solution $(25 \mathrm{mg} / 100 \mathrm{cc}$ acetone) for $10 \mathrm{~min}$. Finally, the plate was again washed with acetone and then dried under a stream of warm air (about $40^{\circ} \mathrm{C}$ ) (by a hair dryer or in a hot air oven) for $2-5 \mathrm{~min}$. Blowing with hot air, up to $80^{\circ} \mathrm{C}$ for 10 min did not change the fluorescence intensity appreciably.

Detection of fluorescent spot. The fluorescent spot was detected by a long wave length ultraviolet light (UVL 56, Ultraviolet Products, Inc., San Gabriel, Calif., USA). It takes considerable experience to recognize the abnormal pattern from normal. Thus it is advisable that the investigator obtain enough experience with samples from normal persons of all ages as well as from patients with known diseases.

Jpn. J. Human Genet. 


\section{RESULTS}

\section{Deproteinization}

Of the amino acids tested, all showed maximum adsorption to Dowex within 10-20 min. These adsorbed amino acids can be eluted into the $\mathrm{NH}_{4} \mathrm{OH}$ supernatant in about $40 \mathrm{~min}$. A typical kinetics of adsorption and elution of an amino acid is shown in Fig. 1 and the results of various amino acids tested are summarized in Table 2. Determination of protein concentration by Lowry method (Lowry et $a l ., 1951)$ before and after Dowex treatment revealed no appreciable loss of protein, indicating that the selective adsorption of amino acids by Dowex is a very effective way of deproteinization. It should be pointed out that the deproteinization step results in an increase in amino acid concentration 1.5 to 3 times for most amino acids (Table 2), a step particularly useful for serum, as most of the amino acids concentrations are below the level of detection. Deproteinization procedure, however, decreased the resolution of amino acids significantly. After the Dowex treatment, a bluish fluorescent area was created which ran across the plate between Tyr and Gly. Fortunately, there is no significant aminoacidopathy with amino acid(s) having the mobility in this area.

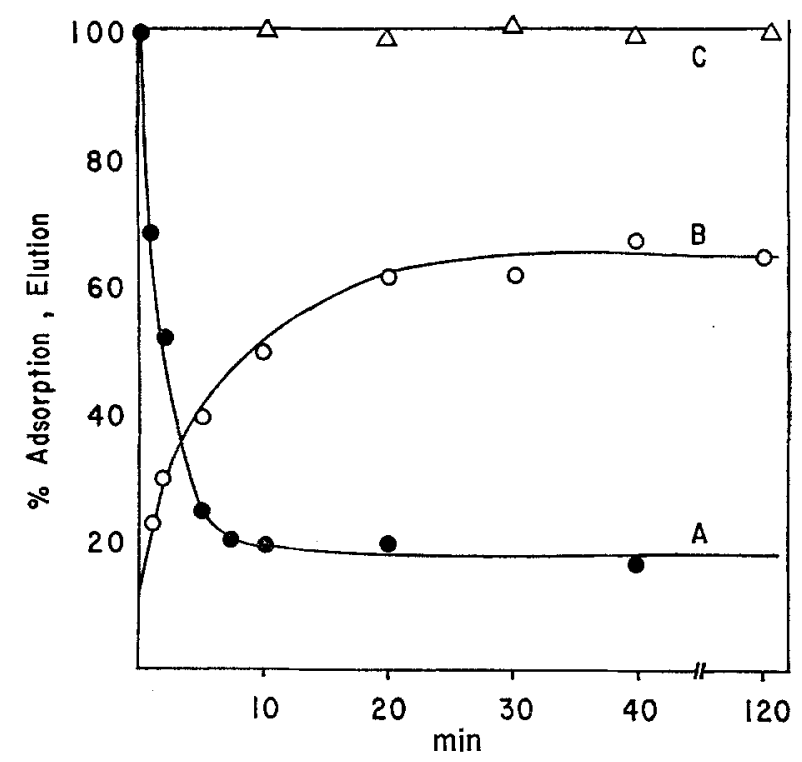

Fig. 1. Kinetics of the deproteinization of serum as determined by $\left[{ }^{14} \mathrm{C}\right]$ valine. $\triangle$, protein remains in the supernatant; 9 , adsorption of $\left[{ }^{14} \mathrm{C}\right]$ valine to the Dowex; $O$, elution of $\left[{ }^{14} \mathrm{C}\right]$ valine to the supernatant. For detail, see METHOD. 
Table 2. Deproteinization of serum and urine. Adsorption and elution were performed as described in Fig. 1. After 20 min of adsorption, the percentage of adsorption by the

Dowex was calculated by subtraction of the radioactivity seen in the supernatant from the original radioactivity. After $40 \mathrm{~min}$ of elution, the percent elution was obtained by recovery of radioactivity in the eluent as compared to the original radioactivity. Extent of purification represents the ratio of radioactivity in eluent and that before the adsorption.

\begin{tabular}{lccccccc}
\hline \multirow{3}{*}{$\begin{array}{c}\text { Amino } \\
\text { acid }\end{array}$} & $\begin{array}{c}\text { Adsorption } \\
(\%)\end{array}$ & $\begin{array}{c}\text { Elution } \\
(\%)\end{array}$ & $\begin{array}{c}\text { Purifica- } \\
\text { tion }\end{array}$ & & $\begin{array}{c}\text { Adsorption } \\
(\%)\end{array}$ & $\begin{array}{c}\text { Elution } \\
(\%)\end{array}$ & $\begin{array}{c}\text { Purifica- } \\
\text { tion }\end{array}$ \\
\hline Arg & 86.3 & 26.0 & 1.02 & & 90.1 & 26.7 & 1.05 \\
Asp & 89.4 & 65.0 & 2.55 & & 76.8 & 47.4 & 1.86 \\
Cys & 85.8 & 45.0 & 1.77 & & 96.5 & 53.4 & 2.10 \\
Gly & 96.8 & 64.5 & 2.72 & & 88.3 & 49.9 & 1.96 \\
His & 87.0 & 47.1 & 1.85 & & 86.2 & 48.9 & 1.92 \\
Leu & 95.4 & 61.9 & 2.43 & & 89.2 & 55.3 & 2.17 \\
Lys & 98.0 & 67.1 & 2.63 & & 98.6 & 52.2 & 2.05 \\
Met & 73.4 & 42.6 & 1.67 & & 72.9 & 48.1 & 1.89 \\
Pro & 96.9 & 68.1 & 2.67 & & 87.9 & 58.1 & 2.28 \\
Phe & 74.3 & 41.6 & 1.63 & 87.4 & 44.9 & 1.76 \\
Tyr & 72.9 & 39.1 & 1.53 & 72.6 & 36.0 & 1.41 \\
Val & 80.6 & 50.6 & 1.94 & 72.0 & 42.4 & 1.66 \\
\hline
\end{tabular}

\section{Supporting matrix}

Many types of commercially available thin layer plates were tested, including polyamide sheets, silica gel, aluminum oxide, plain cellulose and various chemically modified cellulose. Of those tested, the plain cellulose plate produced the best resolutions of amino acids with the least diffusions (we did not compare the cellulose plates from various companies). Although microcrystal cellulose thin layer plates were available, we found that they not only failed to improve the resolution, but also took a longer period for the chromatography.

Before chromatography, $0.1 \mu l$ of sample gave a spot of about $2 \mathrm{~mm}$ in diameter. After the run, the fluorescent spot was about 3-4 mm, even for those amino acids with high mobilities. Therefore, diffusion of the sample in this matrix during the chromatography is minimal.

\section{Chromatographic result}

The amino acids, with the exception of proline which appeared as a non-fluorescent dark spot, showed as spots with bright yellowish fluorescence. It increased in intensity in the first $2 \mathrm{hr}$, stayed the same level for 1-2 days and then gradually faded. The dark proline spot, however, faded within $2 \mathrm{hr}$. Exposure of the plate 
to the fumes of $\mathrm{HCl}$, ammonia, dioxane or triethylamine did not result in appreciable improvement in the intensity of fluorescence.

The chromatographic pattern of amino acids was in general agreement with those reported with paper chromatography using the same solvent system (Fig. 2). In the order of decrease in mobility, they are Leu/lle, Phe, Met/Val/Trp, Tyr, Pro, $\mathrm{Ala} / \mathrm{Thr} / \mathrm{Glu}, \mathrm{Gly} / \mathrm{Gln} / \mathrm{homocystine,} \mathrm{His/Lys/Arg,} \mathrm{and} \mathrm{Cys.} \mathrm{As} \mathrm{in} \mathrm{an} \mathrm{ordinary} \mathrm{one}$ dimensional paper chromatography, some amino acids do not separate well from each other (for example, Met, Val, and Trp). Leu and Ile have tendencies to diffuse. The spots were less well defined and appeared to fade faster. Val, though showing similar tendency to diffuse, diffuses to a lesser extent. Figure 3 shows urinary amino acid patterns of various ages and diseases.

The protein in the urine is usually in a relatively low concentration that no or little streaking effect is observed in the chromatography. Deproteinization of urine prior to the chromatography is usually unnecessary (unless urine contains large amounts of protein as in patients with nephrotic syndrome).

Deproteinization of serum is essential for several amino acids. A large quantity of serum protein causes a strong fluorescent streak covering lower half of the chromatogram and makes the identification of amino acids in this area impossible (Fig. 4). Amino acids with mobilities higher than that of glycine (Leu, Ile, Phe, Val, Tyr) (Fig. 2) are unaffected. The recoveries of these amino acids were not affected by the presence of proteins (Lee et $a l ., 1983$ ). Therefore, if amino acids to be studied are one of the above, for example, the serum of PKU patient, depro-

\section{- Solvent front}

$$
\begin{array}{ll}
\text { O Leu/Ile } & \text { Lhe } \\
\text { Phe } & \text { Met/Val/Trp } \\
\text { Oyr } & \text { Tyr }
\end{array}
$$

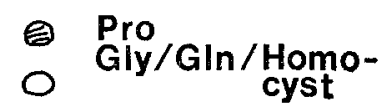

$\diamond$ His/Lys/Arg

- Cystine

- Origin

Fig. 2. Chromatographic pattern of amino acids. 
teinization is unnecessary. It is worth mentioning that normal serum, whether deproteinized or not, contains much less fluorescent spots than those from urine (Fig. 4).

\section{Sensitivity}

There was considerable difference in the sensitivity with different amino acids. The majority of them can be detected with concentration of $0.25 \mathrm{~mm}$ or greater, though some need about 30-60 min to attain the maximal fluorescence. In general, amino acids can be divided into four groups according to the minimum amount required for detection:

(I) $0.25 \mathrm{~mm}$, immediately after staining-Leu, Ile, Phe, Met, Tyr, Gly, His and homocystine.

(II) $0.25 \mathrm{~mm}, 30 \mathrm{~min}$ after staining-Lys, Trp, Arg, Ala, Ser, Thr and cystine.

(III) $0.5 \mathrm{~mm}$ or above, $30 \mathrm{~min}$ after staining-Val, Asp, Glu and GIn.

(IV) $5.0 \mathrm{~mm}$ or above, immediately after staining-Pro.

Therefore, it is advisable to read the plate immediately after staining and 30-60 min after the staining.

Urine from patients or artificially prepared samples of PKU (type I), MSUD, NHT, cystinuria (type I), ketotic and non-ketotic hyperglycinemia (Table 1) were detected without difficulty. Cystinuria is the easiest to be detected due to the drastic increase of cystine and dibasic amino acids. We, however, frequently failed to detect homocystinuria and histidinuria. Rare amino acid disorders such as hypervalinemia, hyperprolinemia, hyperlysinemia, and urea cycle abnormalities were not tested in this study.

Serum from patients with PKU was also detected readily. Serum from other types of diseases were not tested. The detectability of these diseases remained to be investigated.

\section{DISCUSSION}

We reported here a rapid, sensitive, simple, inexpensive micro technique for detection of amino acids in urine and blood. The method requires only $0.1 \mu 1$ of sample, uses commercially available thin layer plates, and takes less than $1 \mathrm{hr}$ to perform. No special facility or equipment is needed.

Great caution must be exercised in the interpretation of the results, particularly that from urine. Urinary amino acid levels are greatly influenced by age, especially during the first few months of life (Bremer et al., 1981), mainly due to the maturation of enzymes involved in the metabolism and renal tubular reabsorption system. Using urine as the test sample however, had several advantages including one that no venous puncture is necessary, and thus, samples could be collected by the parents.

An important step in correcting the variables due to age is to correlate the value 


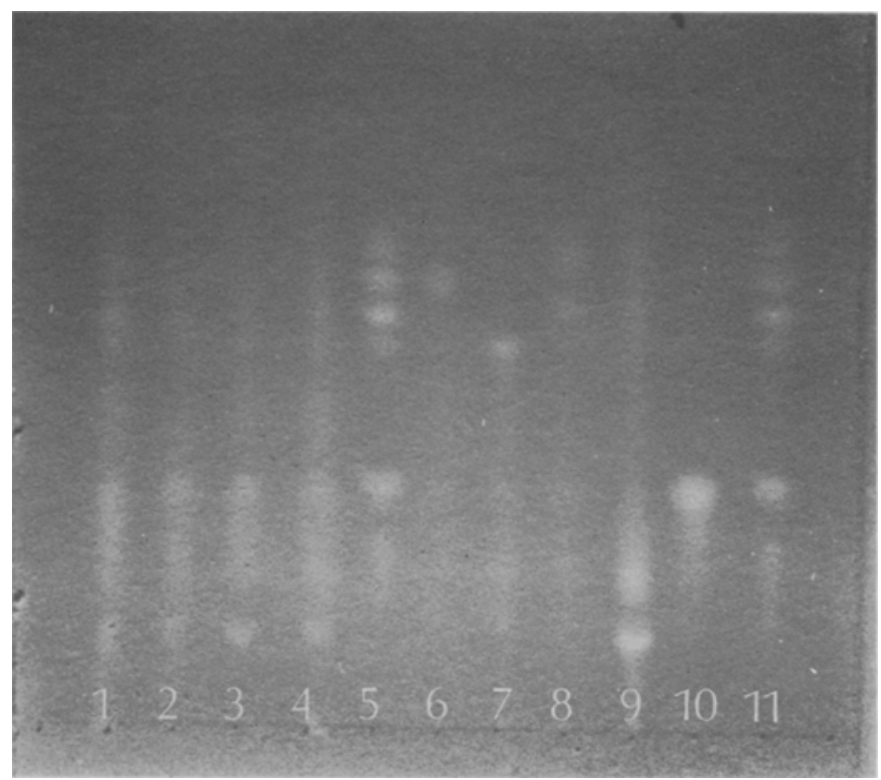

Fig. 3. Chromatographic pattern of urine: 1 , newborn; 2, age 3 months; 3, age 1 year; 4, adult; 5 and 11, standard amino acid mixture (see MeтноD); 6, PKU patient; 7, NHT; 8, MSUD; 9, cystinuria; 10, hyperglycinuria.

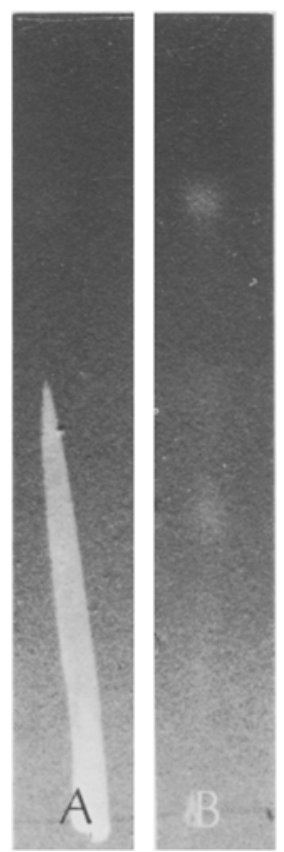

Fig. 4. Chromatographic pattern of PKU serum without (A) or with (B) deproteinization.

Vol. 28 , No. 4, 1983 
to a reference compounds or parameter such as body surface or creatinine content, the latter is in itself dependent, to some extent, on the protein intake. In the method presented here, we did not make such corrections. This is because there is usually a great difference between the level of amino acid in normal individuals and those with aminoacidopathies, frequently, more than a 10-fold (Table 1). The correction of the above parameters probably does not significantly alter the outcome (fluorescent positive or negative). Nonetheless, we recommend that this method is used primarily for patients older than one year, and that caution should be exercised when present method is applied to subjects younger than this age.

While our method can readily detect conditions such as MSUD, PKU, NHT, hyperglycinemia, cystinuria from urine samples, we have difficulty in identifying the abnormal concentrations of amino acids in the low mobility region, such as those from histidinuria. Sensitivity of our technique is enough to detect most of the amino acids with concentration greater than $0.25 \mathrm{~mm}$ (most of the abnormal amino acid has urinary amino acid concentration exceeding $1.0 \mathrm{~mm}$, Table 1), but difficulty arises from the fact that normal urine contains numerous fluorescent positive spots in the middle and lower part of the chromatogram. Two important aminoacidurias, homocystinuria and histidinemia, thus escape detection. Homocystine has mobility similar to that of glycine which is the most abundant amino acid in the urine. Therefore, an increased homocystine is masked by the presence of glycine. Although a patient with homocystinuria will most likely have increased Met (up to $0.4 \mathrm{~mm}$ ) the increase is usually not high enough to be readily detected by this method. Fortunately, homocystinuria is a rare condition, 1 in 230,000 (Levy, 1973). If homocystinuria is strongly suspected, a two dimensional chromatography of the same plate followed by the same staining technique can be applied.

Although urinary His concentration in histidinemia is high enough to be detected by our method, urine from normal persons also contain large amounts of His (Table 1). Thus it is frequently difficult to distinguish the normal from the abnormal. The fact that clinical significance of histidinemia is probably minimal (Ghadini, 1981) makes the detection of histidinuria less urgent.

Arg, Lys and cystine also have low mobility in this solvent system (lower third of the chromatograms, Fig. 2). Arg and Lys for some unknown reasons, have a tendency to streak, thus making identification of individual amino acid difficult. In case of cystinuria, because of its general and overwhelming increase in cystine, the detection of this condition has been the easiest.

Although normal urine contains large amounts of glycine resulting in an intense fluorescent spot, patients with ketotic or non-ketotic glycinuria still can be detected by present method. In these conditions, the glycine excreted in urine is so great in quantity (Table 1), that the increase in intensity is still appreciable. The abnormal quantity of glycine can be confirmed by chromatography of a diluted urine (10-20 fold). With normal urine glycine spot will become very faint or invisible, while urine from the above conditions still yield a strong fluorescent spot. 
It should be mentioned that we have not carried out studies to examine the extent of false-negative and/or false-positive in our technique. Therefore, at this time, the method should not be used as a sole detecting method, but rather, as a preliminary screening technique. This technique can be used as an adjunct study for conditions such as PKU, MSUD, NHT, hyperglycinemia and cystinuria. In the work-up of these conditions, our method can replace the conventional paper chromatography technique.

We can further foresee several advantages for our method:

1. The method can be readily adapted to a screening program. With a plate holder (used in clinical laboratory), in a standard staining jar, thirty $7.5 \mathrm{~cm} \times 6.0 \mathrm{~cm}$ plates (each with 10 samples) can be stained simultaneously within half an hour. Thus, this technique can be used in many developing or underdeveloped areas where sophisticated laboratory facilities are not available.

2. The amount of urine needed is extremely small: $0.1 \mu 1$. Thus, the time needed to collect the sample can be greatly reduced. If necessary, the sample can be directly obtained from the diaper with a microcap tube.

3. Since the assay can be finished within $1 \mathrm{hr}$, this method can provide critical information for the management of urgent clinical conditions. For example, if a patient has clinical signs suggestive of MSUD, this quick qualitative test can provide a preliminary result within $1 \mathrm{hr}$ instead of waiting for the amino acid analysis which may take a day or more. Thus, treatment can be initiated more quickly.

4. In the screening of PKU many of the hyperphenylalaninemias are not due to the classic PKU (type I). In many instances it is associated with increase in tyrosine concentration. In the ordinary PKU screening program, the concentration of tyrosine has to be determined separately. In our fluorescamine test both the phenylalanine and tyrosine will appear simultaneously in the same plate. Therefore, it is possible that hyperphenylalanine can be differentiated from the beginning. Similarly, simultaneous increase of dibasic amino acids in cystinuria can be identified in the same chromatography.

5. As demonstrated from our laboratory (Lee et al., 1983), it is possible to extend our method to roughly estimate amino acid concentrations in the urine or serum. This rapid semi-quantitative estimation will greatly facilitate the management of patients such as PKU patients under dietary restriction.

Acknowledgement The authors would like to thank Dr. C.Y. Lai for his reading and criticism of manuscript.

\section{REFERENCES}

Ambler, R.P. 1972. Enzymatic hydrolysis with carboxypeptidases. In Methods in Enzymology, Hirs, C.H.W., and Timasheff, S.N., eds., Academic Press, Inc., New York, Vol. 25-B, pp. 143154.

Bremer, S., Duraan, M., Kamerling, J.P., Przyrembel, H., and Wadman, S.K. 1981. Disturbances of Amino Acid Metabolism: Clinical Chemistry and Diagnosis, Urban \& Schwarzenberg, Munich, W. Germany, section C. 
Felix, A.M., and Jimenez, M.H. 1974. Usage of fuorescamine as a spray reagent for thin layer chromatography. J. Chromatogr. 89: 361-364

Ghadini, H.K. 1981. Histidinemia, biochemistry and behavior. Am. J. Dis. Child. 135: 210211.

Kelly, S. 1977. Biochemical Methods in Medical Genetics, Charles C. Thomas Publishers, Springfield, 111., pp. 17-25.

Lai, C.Y. 1977. Detection of peptides by fluorescence methods. In Methods in Enzymology, Hirs, C.H.W. and Timasheff, S.N., eds., Academic Press, Inc., New York, Vol. 47-E, pp. 236-243.

Lee, M.L., Toke, D., and Wang, T.R. 1983. A rapid, semi-quantitative determination of phenylalanine, glycine and cystine from urine and serum. Jpn. J. Human Genet. 28: 285-289.

Levy, H.L. 1973. Genetic screening. In Advances in Human Genetics, Harris, H. and Hirschhorn, K., eds., Prenum Publishing Corp., New York, Vol. 4, pp. 1-104.

Lowry, O.H., Rosebrough, N.J., Farr, A.L., and Randall, R.J. 1951. Protein measurement with the folin phenol reagent. J. Biol. Chem. 193: 265-275.

Stein, S., Bohlen, P., Stone, J., Dairman, W., and Udenfriend, S. 1973. Amino acid analysis with fluorescamine at the picomole level. Arch. Biochem. Biophy. 155: 203-212

Thomas, G.H., and Howell, R.R. 1973, Selected Screening Tests for Genetic Metabolic Diseases, Year Book Medical Publishers, Inc., Chicago, Ill., pp. 81-96

Udenfriend, S., Stein, S., Bohlen, P., Dairman, W., Leimbruber, W., and Weiele, M. 1972. Fluorescamine: A reagent for assay of amino acids, peptides, proteins and primary amines in the picomole range. Science 178: 871-872 